\title{
Histoire et épistémologie des savoirs locaux et autochtones
}

\author{
De la tradition à la mode
}

History and Epistemology of Local and Indigenous Knowledge : from Tradition to

Trend

\section{Marie Roué}

\section{CpenEdition}

\section{Journals}

Édition électronique

URL : http://journals.openedition.org/ethnoecologie/813

DOI : 10.4000/ethnoecologie.813

ISSN : 2267-2419

\section{Éditeur}

Laboratoire Eco-anthropologie et Ethnobiologie

\section{Référence électronique}

Marie Roué, «Histoire et épistémologie des savoirs locaux et autochtones », Revue d'ethnoécologie [En ligne], 1 | 2012, mis en ligne le 02 décembre 2012, consulté le 19 avril 2019. URL : http:// journals.openedition.org/ethnoecologie/813; DOI : 10.4000/ethnoecologie.813

Ce document a été généré automatiquement le 19 avril 2019

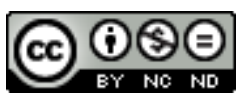

Revue d'ethnoécologie est mis à disposition selon les termes de la licence Creative Commons Attribution - Pas d'Utilisation Commerciale - Pas de Modification 4.0 International. 


\section{Histoire et épistémologie des savoirs locaux et autochtones}

De la tradition à la mode

History and Epistemology of Local and Indigenous Knowledge : from Tradition to

Trend

Marie Roué

\section{Introduction}

1 Si l'intérêt académique pour les savoirs autochtones, indigènes en anglais et en espagnol, date de quelques dizaines d'années, ils existent, quant à eux, depuis plusieurs millénaires. Les sociétés qui les ont développés n'ont pas attendu une reconnaissance officielle pour tirer parti des plantes et animaux ou les domestiquer. Encore aujourd'hui une grande partie de la subsistance et de la pharmacopée dans le monde est acquise grâce aux savoirs et savoir-faire des chasseurs-cueilleurs, pêcheurs ou agriculteurs. Pourtant le statut de ces savoirs, en particulier leur reconnaissance et leur légitimité dans le domaine de la gestion, comme support de décisions dont la société et les scientifiques reconnaîtraient le bien-fondé, est une source de débats sans cesse renouvelés.

2 Nous poserons ici la question de l'origine de la notion de «savoirs locaux » avant son apparition sur la scène politique et médiatique. En remontant aux sources nous visons deux buts qui se conjuguent. Nous voulons tout d'abord informer le lecteur qui, en particulier en France, n'a eu accès à cette notion que depuis qu'elle est devenue un des piliers du politiquement correct, et qu'elle est utilisée de façon un peu superficielle, au même titre que d'autres notions à la mode: "développement durable", "recherche participative ", par tous les acteurs pour rendre leur discours plus actuel. Nous espérons également rendre sa crédibilité scientifique à une notion qui, depuis son adoption par plusieurs conventions internationales, fait l'objet d'un engouement parfois simplificateur, et est souvent dévoyée jusqu'à la caricature. Notre démarche se conjugue en deux temps. Dans une première partie épistémologique nous analyserons les tensions et enjeux autour 
de cette notion dans les cinquante dernières années. Nous proposerons ensuite une brève histoire de cette notion en présentant les textes fondateurs qui ont permis à cette notion issue des travaux de biologistes et d'anthropologues d'émerger.

\section{Définir les savoirs « autochtones »}

3 Définir les savoirs indigènes n'est pas chose facile. Avant même de nous interroger sur ce qu'ils sont, il nous faut comprendre les enjeux et conflits autour de leur dénomination. En effet nul ne s'entend sur le choix des termes, ou plutôt tous s'accordent à juger toutes les désignations autres que celles qu'ils préconisent insatisfaisantes.

\section{Comment les nommer : savoirs traditionnels, écologiques, autochtones, ou locaux?}

Doit-on parler de savoir écologique traditionnel (Traditional Ecological Knowledge, TEK), ou de savoir traditionnel (Traditional Knowledge, TK), comme on l'a fait dans les années 80 au tout début du développement de ce champ et comme certains le font toujours?

5 Serait-il préférable d'abandonner cette désignation au profit d'une dénomination plus politisée, celle de savoir autochtone (Indigenous Knowledge, IK)?

6 Doit-on tout au contraire choisir une notion la plus neutre, celle de savoirs locaux (Local Knowledge, $L K)$ ? Si cette dernière dénomination a l'inconvénient d'être si large qu'elle en arrive à signifier tout et son contraire, elle a au moins l'avantage de n'exclure aucun des détenteurs de ces savoirs, et en particulier tous ceux qui ne sont pas autochtones ou ne veulent pas être désignés comme tels.

7 L'avantage des premières désignations (TEK ou TK) était d'évoquer immédiatement l'ancienneté de ces savoirs, et d'annoncer clairement leur domaine, celui de la nature, en posant d'emblée la question du rapport entre les savoirs des populations locales avec la science de l'écologie. Pourtant cette «scientisation» des savoirs autochtones a aussi ses inconvénients. Il est réductionniste de désigner ces savoirs du nom d'une discipline scientifique, alors qu'ils sont également des savoir-faire, un mode de vie, une représentation du monde et une éthique. Ce que savent les peuples autochtones, ce qu'ils savent faire et surtout leurs représentations du monde sont intimement liées: leur pensée procède d'une démarche holiste. La vision occidentale dichotomique qui sépare la Nature de la Culture, et l'homme de tous les autres êtres vivants, n'est nullement la leur. Les peuples autochtones ne font jamais de la science en tant qu'activité séparée. Notre science occidentale, elle, s'est constituée en se coupant de la philosophie et de la religion, et en séparant de surcroît chaque domaine scientifique de celui des autres sciences. Enfin l'opposition entre savoir et croire, cette volonté d'universalité de la science qui cherche des lois valables en toutes circonstances est une démarche que ne partagent qu'en partie les « savants » autochtones.

\section{Tradition et contemporanéité}

8 Un autre concept de l'appellation TEK pose problème et suscite des conflits stériles. C'est celui de tradition. Considérer comme traditionnelles des sociétés qui, il n'y a pas si longtemps, étaient appelées primitives ou sauvages, pourrait en effet être suspecté d'un 
parfum de darwinisme social. $\mathrm{Si}$, comme on a tendance à le comprendre dans un système d'oppositions binaires, traditionnel signifie ce qui ne change pas, ce terme serait inadéquat. Les savoirs traditionnels deviendraient alors au mieux des survivances, au pire des anachronismes, dont la conservation engendrerait une folklorisation et une muséification de ce qui est encore vivant.

La tradition doit être comprise au contraire comme ce qui relie les hommes d'aujourd'hui aux hommes d'hier, c'est-à-dire l'interprétation par des sociétés contemporaines de ce qu'elles ont reçu de celles qui les ont précédées. Dans cette acception la tradition n'exclut pas le changement. (Amselle 2008 ; Goody 1977 ; Lenclud 1987 ; Pouillon 1998). Les peuples autochtones, tout comme les cultures occidentales, sont en effet modernes et vivent, eux aussi, dans la contemporanéité (Latour 1991 ; Augé 1994). Quand les Inuit adoptent, pour chasser, tous les derniers avatars techniques de nos sociétés, vêtement comme mode de transport ou arme à feu, ils continuent à chasser «leurs " animaux, en utilisant leur compétence du terrain, leurs savoirs sur les conditions climatiques, les déplacements, la biologie des animaux, et en considérant, tout comme leurs ancêtres, qu'il leur faut respecter les animaux qui se donnent à eux. Cette acception de la tradition est donc bien loin d'une opposition à la modernité.

10 Revendiquer aujourd'hui «sa » tradition est un acte réflexif d'une société qui s'interroge sur elle-même et sur son changement rapide. Les pêcheurs bretons, tout comme les Samis, ne veulent plus être vus comme des victimes passives d'un monde qui les contraint à devenir semblables à tous en devenant "modernes ». L'anthropologie a heureusement abandonné le concept d'acculturation qui faisait florès dans les années 60 , et imaginait les autochtones toujours perdants face à l'occidentalisation qui les guettait et gagnait à tous les coups. On parle aujourd'hui d'« agencéité » : les autochtones sont les acteurs de leur destin et utilisent leurs stratégies propres, leurs ressources pragmatiques et cognitives pour choisir de recomposer à leur guise leur devenir. De nombreuses pratiques et savoirs comportent une part de créolisation comme les intellectuels des Antilles françaises, las de la négritude de Césaire, le revendiquent (Glissant 1997). Loin d'être un héritage, la tradition devient une revendication dans un monde où la globalisation n'implique plus l'uniformité ou l'homogénéisation.

\section{Indigènes et autochtones ou locaux?}

11 L'étiquette "savoirs autochtones » (IK, le terme indigenous étant traduit en espagnol par indígeno mais en français par « autochtone ») a l'avantage d'être acceptée par quatre cents millions d'autochtones. Lors de sa septième session en 2008, le Forum Permanent sur la Question Autochtone revendiquait en tant que peuple autochtone d'être la troisième nation au monde par le nombre. Depuis 2002, le Forum Permanent sur les questions autochtones ${ }^{1}$ siège à New York aux Nations Unies et traite, en particulier, de la question des savoirs et des droits de propriété. La Déclaration sur les droits des peuples autochtones a été adoptée à New York le 13 septembre 2007 par l'Assemblée générale des Nations Unies (ONU) après vingt ans de négociations à 143 voix contre 4 (des États-Unis, du Canada, de l'Australie et de la Nouvelle-Zélande) et 11 abstentions. Elle affirme les droits à réparation et à l'autodétermination pour les 370 millions d'autochtones dans le monde et confirme la reconnaissance internationale des populations autochtones et leur présence sur l'échiquier géo-politique international. 
12 Pourtant si nous décidons de parler de "savoirs autochtones", en anglais IK, nous tombons dans un autre piège sans fond: qui est autochtone et qui ne l'est pas? De nombreux anciens colonisés, en particulier dans la zone d'influence francophone, ne tiennent nullement à conserver l'étiquette infamante d'« indigène » que les oppresseurs allochtones utilisaient à leur égard lors d'une période révolue, la colonisation. Rappelons qu'en France le code de l'indigénat, adopté en 1881 puis étendu à l'ensemble des colonies françaises en 1889, n'a été aboli qu'en 1945 et s'est appliqué jusqu'à l'indépendance de l'Algérie. Il distinguait les citoyens français des « sujets français ", ces « indigènes » étant passibles de toutes sortes d'interdictions et de privations de la liberté en cas de rébellion contre leur colonisation. On ne s'étonnera donc pas que la plupart des peuples francophones préfèrent parler de savoirs paysans ou locaux. D'autant plus que comme les Africains ou les Asiatiques le soulignent, chez eux tous les peuples de leur continent sont en quelque sorte "d'origine ", c'est-à-dire indigènes ou autochtones. Il n'y a pas eu, comme dans le cas des colonisations américaines ou australiennes, une première population autochtone, suivie d'une deuxième vague venue d'ailleurs qui a dominé la première. Pour de nombreux ex-colonisés l'imposition de l'identité « indigène » apparaît comme un nouvel affront, venu cette fois de la domination des Indiens d'Amérique dans l'arène autochtone.

Reste enfin la question plus générale, hors du refus de l'indigénat post-colonial que nous venons de décrire, de la définition de ce qu'est un peuple indigène ou autochtone. Le principe adopté dans un rapport de l'ONU de $1983^{2}$ sur la prévention de la discrimination et la protection des minorités reconnait «le droit des peuples indigènes à définir euxmêmes qui est, et ce qui est, indigène ». Outre l'auto-identification, les critères généralement admis pour définir l'autochtonie sont la qualité de descendants de populations qui étaient les premières à habiter les lieux, une identité ethnique distincte et la vulnérabilité. Il s'agit bien là d'une définition politique, qui permet à des populations faisant face localement à un État qui ne reconnaît généralement ni leurs droits territoriaux, ni par conséquent le droit de vivre selon leur mode de vie propre, de revendiquer leur différence et leur identité commune. Selon le WGIP (Working Group of Indigenous Peoples) du forum des Nations Unies, 300 à 400 millions de membres des peuples indigènes dans le monde appartiendraient à 5000 groupes dans plus de 70 pays, l'Asie ayant la population autochtone la plus importante. La question de l'identité s'exprime en dernière analyse à travers un choix : qui peut être autochtone et qui ne veut pas l'être ?

C'est donc pour englober tous ceux qui détiennent un savoir ancré dans une tradition que l'on parle de savoir local ou de savoirs locaux. Cette dénomination permet de prendre en compte les paysans, marins et autres locaux qui détiennent des corpus de savoirs sur leur environnement naturel sans pour autant se considérer comme, ou être, une population autochtone. Le programme LINKS (Local and Indigenous Knowledge Systems) de l'UNESCO', lancé en 2002, parle pour sa part de "systèmes de savoirs locaux et autochtones ». L'ajout $\mathrm{du}$ mot "systèmes", qui est depuis fréquemment repris, est une revendication du caractère systémique de ces savoirs, trop souvent décrits par leurs détracteurs comme un ramassis de connaissances éparses et anecdotiques, par opposition à la science.

\section{Légitimité des savoirs locaux et cogestion}

15 La reconnaissance et la légitimité des savoirs locaux et autochtones permettent en effet aux détenteurs de ces savoirs de sortir de l'humiliante domination qu'a exercée l'État, par 
l'entremise de la science officielle. Elle permet également de sortir du conflit qui se déroule dans de nombreuses nations autour de la propriété des terres ancestrales des populations autochtones. Peu de peuples détiennent les titres de propriété de leurs terres au sens où l'entend notre droit occidental, même s'ils en sont les premiers et très anciens occupants, et continuent à les utiliser. Mais les droits d'usage ont le défaut d'être peu contraignants lorsque plusieurs utilisateurs se les disputent. Au Canada par exemple, où les TEK ont été mis en avant bien avant le Sommet de la Terre de Rio par des groupes conjoints d'experts scientifiques et d'autochtones, la reconnaissance de ces savoirs locaux a permis aux populations de revendiquer la cogestion de leurs ressources. Sans s'épuiser dans les combats incessants que représentaient les Land Claims, où État et Nations autochtones pouvaient se battre à travers des armées d'avocats pendant des décennies sans arriver le plus souvent à des résultats satisfaisants, ces populations ont trouvé une solution élégante qui permet d'éviter un conflit stérile et sans fin avec l'État majoritaire en revendiquant la reconnaissance de leur savoir et de leur gestion.

On présente parfois, bien à tort, l'avènement des savoirs locaux comme un obscurantisme et comme l'oubli des avancées scientifiques dans le domaine de la biologie, de l'écologie ou des sciences de la gestion. Certains anthropologues, confondant les méthodes des consultants qui, par exemple dans les Rapid Rural Assessment, évaluent en quelques journées ou quelques semaines des situations complexes qui mériteraient un examen plus sérieux des savoirs locaux, parlent même de "populisme méthodologique " (Olivier de Sardan 1991). Soulignons ici que la révolution épistémologique qui concerne les savoirs locaux poursuit les mêmes buts que le mouvement de la démocratie participative (Callon et al. 2001; Charvolin et al. 2007) qui concerne les savoirs profanes. En échangeant dans des forums hybrides, médecins, malades du sida ou parents d'enfants atteints de maladies orphelines participent aujourd'hui à des recherches conjointes qui les concernent. Leurs associations ont lutté pour démontrer que leurs membres peuvent mobiliser d'autres savoirs que ceux des scientifiques. Si les peuples locaux d'un côté, les scientifiques de l'autre, ont, par des méthodes qui ont beaucoup en commun mais divergent sur d'autres points, obtenu des résultats qui concernent le même objet, il est également possible, et même souhaitable, qu'ils puissent travailler en partenariat. Or un véritable partenariat implique une transformation politique des rapports de pouvoir. Savoirs et pouvoirs vont de pair. La domination qui permettait à l'État de prendre des décisions au niveau local pour le plus grand bien des populations, basées sur le seul savoir des scientifiques, n'est plus de mise, même dans nos propres sociétés, a fortiori pour des peuples autochtones. On parle aujourd'hui de démarche participative et de développement durable. Trop souvent pourtant ces approches restent encore une timide rencontre où les uns entendent faire « l'éducation environnementale » des autres.

Dans certains cas pourtant, des programmes de cogestion permettent à un État ou une administration centrale de reconnaître la légitimité des droits des populations autochtones, en particulier celle de gérer les terres qui sont les leurs. C'est ce débat qu'annonçait déjà Johannes dès les années 80 , dans des termes radicaux: comment pourrait-on prétendre que scientifiques et gestionnaires allochtones sauraient mieux gérer que le savoir local un environnement qu'ils ne connaissent pas?

«Imagine people who confidently assume they can best describe and manage the natural resources of an unfamiliar region alone - ignoring local hunters who know every cave and waterhole and the movements and behavior of a host of local animals - overlooking the farmers who know the local soils, microclimates, pests and seasonal environmental changes - disregarding the native fishermen who 
know the local currents and the movements and behavior of the marine life in their waters. Such, historically, has been the custom of most environmental scientists and natural resource managers working in unfamiliar environments. " Johannes (1989) des chasseurs, pêcheurs, fermiers locaux ou autochtones, et déplore que ces savoirs ne soient pas pris en compte par des chercheurs ou des gestionnaires qui ont la naïveté de se croire en terra incognita, se sentant capables de décrire et de gérer des milieux dont ils n'ont aucune connaissance préalable. Elle émane d'un chercheur qui a rédigé un ouvrage fondamental sur les savoirs des pêcheurs de Micronésie (Johannes 1981), plus de dix ans avant la Convention sur la diversité biologique (CDB) de 1992, puis a édité huit ans après un recueil sur les savoirs locaux auprès de l'IUCN (Johannes 1989).

Avant que d'aller à la recherche des précurseurs de la notion de savoirs locaux, il nous faut rappeler l'article 8j de la Convention de la Biodiversité, CDB, premier texte qui a permis depuis la rédaction de cette convention à Rio en 1992 à la notion de savoirs locaux de se constituer en sujet de droit :

« Article 8 - Conservation in situ

Chaque partie contractante, dans la mesure du possible et selon qu'il conviendra:

j) Sous réserve des dispositions de sa législation nationale respecte, préserve et maintient les connaissances, innovations et pratiques des communautés autochtones et locales qui incarnent des modes de vie traditionnels présentant un intérêt pour la conservation et l'utilisation durable de la diversité biologique et en favorise l'application sur une plus grande échelle, avec l'accord et la participation des dépositaires de ces connaissances (...) et encourage le partage équitable des avantages découlant de l'utilisation de ces connaissances, innovations et pratiques. "

\section{Les origines de la notion de savoir local}

Après avoir tenté d'exposer dans une première partie les enjeux et définitions des savoirs locaux, il nous semble important, pour mieux comprendre cette notion, de nous intéresser aux premiers auteurs qui l'ont fondée, avant et après l'invention de cette nouvelle dénomination.

\section{Les précurseurs}

Dans toute recherche de sources, il y a une part d'arbitraire puisque l'on revisite un auteur du passé à la lueur de questions du présent. C'est le cas aussi dans le domaine qui nous occupe. En effet entre les années 50 et aujourd'hui, surtout parmi les anthropologues qui s'intéressent à la nature, un grand nombre de mouvements, ou du moins d'étiquettes, ont été lancés, sans qu'aucun n'ait rallié définitivement dans son camp les uns ou les autres: ethnobotanique, ethnozoologie, ethnoscience, écologie culturelle, ethnoécologie, ethnobiologie, savoirs écologiques traditionnels, savoirs locaux, savoirs autochtones.

Ils se sont succédé, sans jamais disparaitre tout à fait, ressurgissant avec plus ou moins de force dans un lieu ou un autre, ce qui nous interdit de nous contenter d'une datation successive de plusieurs mouvements. Ils ont coexisté, et l'on peut même dire que la plupart de ces postures ont été successivement ou même tout à la fois adoptées par 
certains chercheurs. Une part de la raison de cette multiplicité est aisée à comprendre : être l'auteur, ou au moins le tenant patenté d'une de ces étiquettes, est fort utile pour acquérir un surplus de capital symbolique. Mais, hors de ces considérations stratégiques qui pourraient nous conduire à négliger ces dénominations, se profile une réelle complexité. En effet, le champ interdisciplinaire dans lequel ces recherches se sont épanouies est par essence l'objet de points de vue différents, selon que l'on considère une question ou une autre, en partant d'une discipline ou d'une autre. Cette nébuleuse gagne à être comprise comme un réseau, dont les membres, les chercheurs, connaissent et participent peu ou prou à ces différents noyaux, dans lesquels leur position est plus ou moins centrale. Harold Conklin, notre premier « ancêtre », peut donc, selon les auteurs et les étiquettes, être considéré comme l'origine et l'incarnation de l'ethnobotanique, de l'ethnoécologie, de l'ethnobiologie, de l'ethnoscience ou des savoirs locaux. Ayant été un des premiers à investir le champ interdisciplinaire des savoirs des peuples «traditionnels" sur la nature, il est d'ailleurs logique qu'il se situe à la croisée des chemins que d'autres prendront par la suite. De même, le nom de Lévi-Strauss est associé avant tout au structuralisme et à l'anthropologie sociale. Pourtant un de ses premiers ouvrages a également été fondateur dans le champ des savoirs locaux.

\section{Conklin, l'ancêtre commun}

23 Le premier universitaire qui consacra sa thèse intitulée The Relation of Hanunoo Culture to the Plant World aux savoirs sur les plantes d'un peuple traditionnel et à l'organisation structurée de ce savoir fut Harold Conklin à Yale, en 1954. Il nota chez les Hanunóo des Philippines 1500 termes de catégories pour les végétaux parmi ceux «les plus fréquemment enregistrés au cours de conversations » qui permettent l'identification des plantes, en décrivant leurs parties constitutives et leurs propriétés. Ses données mettent en évidence, non seulement l'étendue du savoir hanunóo, mais également la haute teneur scientifique de leurs préoccupations. Les Hanunóo passent leurs dîners et leurs soirées à discuter de végétaux :

« Topics discussed during and immediately after the evening meal at Parina were systematically and periodically recorded throughout the year. Plants, especially cultivated varieties, were the subject of discussion in 65 per cent of the cases recorded. » (op.cit.: 73)

24 L'anthropologue n'hésite pas à accorder aux Hanunóo une double crédibilité scientifique, de systématiciens et de botanistes économiques.

«In short, the Hanunóo combine the interests of native systematic and economic

botanists » (op.cit. : 180).

Outre cette spécialisation sur les plantes, les Hanunóo classent également les oiseaux, les serpents, les poissons, et groupent en 108 catégories les milliers de formes d'insectes. Un tel savoir est acquis très tôt, puis augmenté pendant toute une vie.

Conklin raconte comment, à sa grande surprise, une petite fille de 7 ans suggéra un matin qu'il lui montre "les images» de l'ouvrage en trois volumes de Brown sur les plantes utiles des Philippines. Elle avait vu l'ethnologue travailler avec les adultes pour identifier les plantes à partir de cet ouvrage, et était vexée de n'avoir pas été, elle aussi, consultée (op.cit. : 176). Elle se mit alors à commenter le livre, donnant pour chaque plante une désignation hanunóo, ou déclarant solennellement qu'elle «n'avait pas vu cette plante auparavant ». Elle accomplit ainsi la performance d'identifier correctement 51 plantes sur 
75, avec seulement deux erreurs. Comme l'illustre le défi de cette très jeune détentrice des savoirs locaux, l'acquisition du savoir local est initiée dès le plus jeune âge.

L'étude de l'utilisation du monde végétal ou animal existait bien avant les années 50, et a continué à exister sous des dénominations composées du préfixe ethno, peuple, suivi d'un qualificatif : ethnobotanique, ethnozoologie, ethnominéralogie... (Barrau 1985). Mais ce que l'ethnoscience apporta de radicalement nouveau, en se penchant sur les savoirs des peuples locaux sur leur nature, c'est une vision de l'intérieur, par opposition à une ethnobotanique ou une botanique économique coloniale conçues d'emblée comme une science occidentale pratiquée à des fins utilitaires chez les peuples colonisés. L'ethnoscience, en étudiant les classifications que ces peuples faisaient du monde vivant, se consacra aux structures de la pensée, à la " grammaire culturelle » recueillie dans la langue des locuteurs. La collecte des taxinomies mettait pour la première fois en valeur, non seulement l'étendue du savoir des peuples concernés, mais également sa nature en la comparant et la rapprochant des taxinomies scientifiques.

\section{Lévi-Strauss : pensée sauvage et science du concret} données chiffrées pour établir que quelques heures par jour suffisaient à de nombreux groupes pour chasser, pêcher ou cueillir tout ce qui était nécessaire à la subsistance du groupe. Quelques années auparavant, l'ouvrage édité par Lee et DeVore (1968) et intitulé Man the Hunter avait réuni un collectif d'anthropologues qui avait écorné l'image de chasseurs misérables victimes de milieux effroyables, déserts glacés ou torrides dont ils tireraient à peine leur subsistance.

Le premier chapitre de La Pensée Sauvage de Lévi-Strauss (1962) intitulé «La science du concret » est consacré aux savoirs indigènes, qu'il qualifie également de pensée mythique.

C'est tout d'abord l'étendue du savoir local que l'auteur met en avant. Un seul informateur séminole identifie deux cent cinquante espèces et variétés végétales, les Indiens Hopi connaissent trois cent cinquante plantes, les Navaho plus de cinq cents, les Subanun des Philippines utilisent plus de mille termes botaniques, les Hanunóo, près de deux mille. Les usages des plantes et des animaux sont également d'une grande sophistication et dénotent observation et systématisation. Les Bouriates distinguent chez une seule espèce, l'ours, sept vertus thérapeutiques distinctes pour la chair, cinq pour le sang, neuf pour la graisse, douze pour la cervelle, dix-sept dans la bile, deux dans le poil, soit pas moins de cinquante-deux usages thérapeutiques pour une espèce chez un seul peuple!

La première portée de cet ouvrage est donc de sortir de l'hypothèse des peuples primitifs et ignorants. La deuxième nous apparaît aujourd'hui plus importante : dépasser la vision d'un savoir uniquement utilitaire destiné à la subsistance, objectif dont on supposait alors qu'il était dans la plupart des cas presque impossible à atteindre.

\section{Utiliser ce que l'on sait ou ne connaître que ce qui est utile ?}

Pourtant dès 1962, Lévi-Strauss, après avoir démontré l'étendue des savoirs de nombreux peuples autrefois qualifiés de primitifs, niait que ce savoir soit tourné exclusivement vers la recherche de résultats utilitaires. Bien au contraire, citant entre autres Speck, il affirme que les taxinomies autochtones ont pour objectif la connaissance en soi. Grand 
connaisseur des Indiens du nord-est des États-Unis et du Canada, Speck a démontré que ces derniers ont développé une véritable herpétologie, même si la classe des reptiles n'offre aucun intérêt économique pour eux et qu'ils ne consomment ni n'utilisent aucune partie de ces animaux. Leur taxinomie comprend pourtant non seulement des termes distincts pour chaque genre de reptiles, mais également pour les espèces et les variétés (Speck 1923).

De même les Pygmées, qui identifient un nombre phénoménal de plantes et d'oiseaux, ont développé une connaissance des habitudes et des mœurs de nombreuses espèces, $y$ compris celles des chauves-souris, qui ne sont pas pour eux des animaux «utiles ». Tous ces exemples puisés chez de nombreux observateurs au sein de diverses sociétés amènent Lévi-Strauss à conclure qu'en fait ce n'est pas l'usage qui amène à la connaissance, mais plutôt le contraire. Une partie seulement de nombreuses expériences inspirées par le goût du savoir ont pu donner des résultats pratiques :

«... Les espèces animales et végétales ne sont pas connues, pour autant qu'elles sont utiles: elles sont décrétées utiles ou intéressantes, parce qu'elles sont d'abord connues. » (Op.cit. : 21)

Si les indigènes des Philippines ont pu acclimater, nommer et classer, et surtout redécouvrir aussi rapidement les mêmes usages médicinaux que ceux qui avaient été développés au Mexique pour des plantes originaires d'Amérique, c'est en raison des catégories cognitives qu'ils avaient développées pour leur propre flore. Avant l'introduction des plantes américaines, ils employaient déjà des feuilles ou des tiges à saveur amère contre les maux d'estomac. Supposant que toute plante introduite pourrait offrir le même caractère, ils ont très rapidement fait des expériences pour vérifier leur hypothèse (Op.cit. : 27, citant R.B. Fox).

«Pour transformer une herbe folle en plante cultivée (...) cultiver sans terre ou sans eau, changer graines ou racines toxiques en aliments ou utiliser cette toxicité pour la chasse... il a fallu une attitude d'esprit véritablement scientifique, une curiosité assidue et toujours en éveil (...) car une petite fraction seulement des observations et des expériences (dont il faut bien supposer qu'elles étaient inspirées d'abord et surtout par le goût du savoir) pouvaient donner des résultats pratiques, et immédiatement utilisables. »

On peut regretter que la postérité ait choisi de se souvenir seulement du terme de bricolage qui, pour certains lecteurs superficiels, souvent anglo-saxons, a masqué la subtilité d'une réflexion épistémologique sur la comparaison entre pensée "rationnelle » occidentale et pensée " sauvage ». Lévi-Strauss qualifie de "bricolage » " une science que nous préférons appeler première plutôt que primitive ». Cette belle analogie avec le bricoleur qui se sert de tout un bric-à-brac, son trésor, pour mener à bien un nouveau projet, par opposition à l'ingénieur, souvent citée de façon anecdotique, a pourtant relégué au second plan les pages précédentes où l'auteur démontre le caractère scientifique du savoir des peuples traditionnels, basé sur la méthode de l'expérimentation.

Ce que Lévi-Strauss avait déduit dès 1962 des observations de plusieurs chercheurs de terrain mérite pourtant que l'on y revienne, car le stéréotype qui consiste à voir dans les savoirs locaux avant tout des savoir-faire ou savoirs liés à des pratiques, n'a jamais été tout à fait balayé. Peut-être n'est-il d'ailleurs pas dû uniquement à l'ethnocentrisme d'observateurs imbus de darwinisme social, impuissants à imaginer que l'Autre puisse exceller sur un terrain qui est le nôtre. Il est sans doute également tributaire du fait que les observateurs rencontrent sur le terrain, quelle que soit l'activité liée à un champ de 
connaissances, un plus grand nombre de simples praticiens que de grands savants. Dans les sociétés traditionnelles, exactement comme dans les nôtres, il y a des gens ordinaires, des spécialistes, et seulement quelques individus exceptionnels qui sont de grands savants, et que l'on rencontre donc statistiquement peu souvent.

De plus si quelques-uns, à travers une vie passionnée par l'acquisition de connaissances, sont devenus de véritables savants, beaucoup de savoirs liés à des pratiques sont détenus par une fraction de la population. En jargon d'anthropologue, on parle alors de l'importance de choisir de bons informateurs, ou de savoirs spécialisés. D'après Brokesha et Myle (1980, cités par Johannes 1989) les jeunes garçons par exemple sont des experts quant aux fruits comestibles sauvages, tandis que les vieilles femmes connaissent les herbes annuelles et les collecteurs de miel connaissent les détails de la floraison de toutes les variétés de fleurs. Est-ce cette spécialisation toute semblable à celle de nos sciences qui nous amène à refuser aux détenteurs de savoirs locaux une pleine reconnaissance du caractère expérimental et systématique de leur méthode?

Chez Conklin, c'est tout un groupe, les Hanunóo, que l'on voyait passer ses nuits à discuter de propriétés et classifications botaniques. Chez Lévi-Strauss, on embrassait l'étendue du savoir humain à travers une vaste fresque comparative qui brossait à grands traits l'étendue des connaissances naturalistes humaines. Milton Freeman et Bob Johannes nous feront pour la première fois rencontrer des savants autochtones qui sont nommés, photographiés, dont on connaît la biographie et parfois même les méthodes d'apprentissage à partir de l'expérimentation.

\section{Les recherches canadiennes sur l'utilisation des territoires en milieu arctique}

Les recherches menées dans les années 70 dans le nord canadien et dirigées par Milton Freeman (1976) sont antérieures aux recherches de Johannes. Malgré leur grande influence sur la recherche canadienne nordique, elles n'eurent pas l'originalité de ce dernier qui, spécialisé dans la gestion des écosystèmes côtiers du Pacifique, fut le premier à s'intéresser aux individus détenteurs des savoirs locaux et à leur acquisition du savoir. Mais elles peuvent revendiquer d'avoir fait école. Elles ont une postérité que l'œuvre de Johannes, beaucoup plus solitaire, atteindra plus difficilement. Un texte récent de Freeman (2011) fait le bilan des 35 dernières années après la parution de son ouvrage initial. On peut voir par exemple l'extraordinaire effervescence qui règne aujourd'hui dans le champ des observations sur le changement climatique comme une descendance de l'œuvre de Freeman. Citons par exemple The Earth is Faster Now édité par Krupnik et Jolly (2002) qui, en réunissant experts scientifiques et locaux dans des ateliers participatifs, a donné lieu à plusieurs articles tout à fait novateurs dans le champ de l'observation du changement climatique.

40 C'est à la demande de l'association nationale Inuit Tapirisat of Canada que le Ministère des affaires indiennes et du Nord Canada donna en 1973 à Milton Freeman la responsabilité de réunir une équipe de chercheurs, d'interprètes et d'enquêteurs, inuit et allochtones, pour recueillir et analyser un corpus de données sur l'utilisation présente et passée du territoire inuit, incluant la mer. Dans un contexte de revendications territoriales qui amenait l'État et les Inuit à se confronter autour de données historiques pour reconnaître ou refuser des droits territoriaux ancestraux contestés, ces recherches furent financées par l'État. La liste des participants couvre plusieurs pages et cette somme 
impressionnante fut éditée en trois volumes grand format (Freeman 1976). Même si l'origine de cette recherche est la demande d'une organisation inuit et qu'elle vise la perception inuit de la relation entre les hommes et la terre, il ne s'agit pourtant pas encore tout à fait d'une recherche sur les savoirs écologiques locaux. Ceux qui sont interrogés sur leur usage du territoire, leur connaissance de ses ressources terrestres et marines, dans leur propre langue, sont bien des Inuit. Pourtant, sauf à la fin du premier volume, on peut regretter que ce travail pionnier ne leur donne jamais la parole directement. Ils font encore l'objet d'une analyse distanciée de géographie humaine qui divise les régions géographiques de façon arbitraire, et n'accorde que peu d'intérêt aux structures sociales ou aux savoirs écologiques locaux. Les résultats sont pourtant, si on les considère à l'aune des années 70 , impressionnants, et ont produit de nombreux chapitres originaux et un ensemble de cartes du mode d'occupation des Inuit sur un immense territoire jouxtant l'Alaska à l'ouest, le Groenland à l'est, le Manitoba au sud, avec une description espèce par espèce du comportement des animaux et de leur intérêt pour chaque groupe inuit, saison par saison.

41 Cet immense chantier, qui dura plusieurs années, exprimait une volonté hors du commun de démontrer l'importance de l'occupation par les peuples autochtones des régions arctiques, de leurs savoirs et savoir-faire sur les espaces et conditions de vie, mais reste pourtant assez classique dans son rendu. Une de ses réussites les plus remarquables concerne le domaine de la cartographie participative. Ces deux tomes produisirent des cartes détaillées de l'utilisation du territoire, espèce par espèce et région par région. Leur rayonnement contribua fortement au développement d'un véritable mouvement canadien de recherche. Centré tout d'abord sur ce que l'on nomme en anglais les «Land Use Studies ", les recherches sur l'utilisation du territoire formèrent une génération de chercheurs novateurs qui se tournèrent par la suite vers l'analyse détaillée des savoirs inuit sur les espèces et les espaces (Brody 1981 ; Nakashima 1993).

Johannes fut cependant le premier à analyser l'acquisition de ce savoir et sa maîtrise par quelques individus, en mettant en valeur le savant local en tant qu'expérimentateur.

\section{Johannes à la rencontre des savants locaux}

Contrairement à beaucoup de scientifiques qui ont omis de mettre en avant leur « informateur $»^{4}$, Johannes dans son ouvrage Words of the Lagoon (1981), «Les mots de la lagune ", reconnaît qu'il doit une grande partie de ce qu'il sait à un homme, Ngirakland 5 . Le portrait qu'il dresse de ce pêcheur, chef du petit village de Ngeremlengui sur l'île de Palau, nous permet de comprendre comment il a acquis son immense savoir sur les poissons. Cet exemple a aussi valeur de modèle. Tous les chercheurs travaillant sur les savoirs autochtones ont rencontré, même s'ils n'en ont pas ou mal rendu compte, ces figures de savants locaux, cités et respectés par leur propre communauté et les communautés avoisinantes. En décrivant avec précision le processus de l'acquisition du savoir de cet homme, Johannes nous donne donc accès à un modèle heuristique.

Né en 1894, Ngirakland avait environ 80 ans quand il rencontra Johannes. Passionné par son sujet, il se souvient avoir écouté depuis l'enfance tout ce que les hommes se disaient quand ils discutaient de pêche, avec le désir de devenir le meilleur pêcheur et d'en savoir plus que quiconque. Après avoir appris tout ce que son oncle pouvait lui enseigner sur la pêche et les poissons, il se tourna vers les autres pêcheurs pour compléter son savoir. 
Déjà il se comportait en véritable expérimentateur scientifique, réfutant ou confirmant ce que ses « informateurs » lui avaient appris :

« Not willing to accept things without proof, he tested what he was told and thereby

came to discard or modify a number of beliefs held widely by other fishermen. »

Mais très vite il ne s'intéressa plus seulement à la pêche. Il commença à observer les habitudes de petits poissons jamais consommés, auxquels un pêcheur ordinaire ne prêtait nullement attention. Son intérêt scientifique de naturaliste s'étendit ensuite aux plantes et à leur floraison, aux oiseaux, aux insectes.

Il mit au point plusieurs expérimentations pour accroître son savoir, transplantant par exemple des bénitiers géants (Tridacna gigas) dans de l'eau peu profonde et surveillant leur croissance pendant trois ans, ou replantant des arbres tout près de sa maison pour observer leur cycle de floraison. En bref, il raisonnait en savant et agissait pour confirmer ou infirmer ses hypothèses en expérimentateur. Un jour que Johannes s'excusait de lui poser des questions si détaillées, il lui fit cette réponse de savant : «Ne vous excusez pas, car beaucoup de choses importantes peuvent être comprises en mettant ensemble de petits détails». Une affirmation moins étonnante lorsque l'on sait qu'elle vient d'un homme capable de décrire le cycle lunaire du frai de 45 espèces de poisson!

Dans son livre Words of the Lagoon, Johannes (1981) montre comment le mode de vie traditionnel des habitants de Palau, qui correspondait au modèle de l'abondance décrit par Sahlins (1976) dans Âge de pierre, âge d'abondance où l'on produit en quantité suffisante tout ce dont on a besoin, est peu à peu détruit par la rencontre avec le modèle de l'économie de marché. Dans le chapitre 5 intitulé «L'éthique traditionnelle de conservation et son déclin ", il cite un dicton de Palau qui dit: "To have is to share" (Johannes $1981: 68$ ).

Avant la seconde guerre mondiale, sous la colonisation japonaise, les habitants de Palau commencent à vendre du poisson pour se procurer les nouveaux biens importés, comme les bateaux à moteur ou les filets. Ils exercent donc une pression accrue sur le milieu récifal qui entraîne rapidement une obligation de compétition entre les pêcheurs. Le changement de technologie fait alors disparaitre la coopération traditionnelle au profit d'une pêche individuelle que permettent les nouveaux filets kesoke. Au fur et à mesure que l'équipement utilisé se fait plus sophistiqué et que son prix monte, les villageois arrêtent le partage. L'on dit alors localement qu'ils ont acquis des «mains impotentes». Ils commencent à échanger biens et services entre eux seulement pour de l'argent : le poisson devient un bien (commodity) au lieu d'être un facteur de partage social.

Après la Seconde Guerre mondiale et la destruction de l'économie japonaise, Palau est contraint de revenir à un mode de vie traditionnel. Les populations de poisson récifales se rétablissent alors pendant quelques années, pour décliner à nouveau quand les échanges monétaires et commerciaux reprennent. À partir des années 60 , le poisson est une ressource tellement rare dans les eaux territoriales de Palau qu'il est remplacé par l'achat de maquereau en conserve en provenance du Japon. Dès 1973 Palau a totalement perdu son autosuffisance : sa dépendance se traduit par une importation de 82 livres de viande et de poisson en boîte par personne et par an, sans compter le riz. Dans les années 70 la quantité de poissons importés excède le poids de poissons exportés (Johannes 1981: 69 et suivantes). Enfin dans les années 70, même si les chefs traditionnels ont alors parfaitement conscience du caractère non durable de l'exploitation des ressources en raison de leur surexploitation, en particulier quant aux poissons et aux pigeons ou aux forêts, ils ne peuvent y remédier car leur pouvoir a été érodé par les pouvoirs coloniaux 
successifs. Il leur fut donc impossible, même si certains le tentèrent, de rétablir l'éthique et les règles de conservation qui avaient prévalu à Palau avant « les temps modernes ».

\section{Johannes, un pionnier}

Quand Johannes disparut, au début des années 2000, ceux qui l'avaient connu réunirent des témoignages éloquents sur la portée de son œuvre, sous l'instigation de son collègue et ami Kenneth Ruddle, dans le bulletin Ressources marines et traditions (SPC 2003) qu'il dirige.

Le témoignage de Charles Birkeland (2003) (Département de Zoologie, Université d'Hawai) souligne à quel point le fait d'écouter des pêcheurs locaux en les considérant comme des pairs, dont le savoir local était complémentaire de celui des scientifiques, était original et pionnier au moment où Johannes le fit :

«His pioneering work was exceptionally original and influential because he followed his own insight into truly important processes and was not constrained by the mainstream paradigms and by the perspectives of his administrators (...) $\mathrm{He}$ observed that Pacific Islanders knew their coral reef fishes far better than scientists, and that their traditional management practices were more effective. He was able to listen to local fishermen, placing their knowledge as complementary to science, rather than patronizing them with his technical training. It really took an open mind to be the first to listen to uneducated people as peers. "

\section{Conclusion}

Ce qui pourrait nous surprendre c'est que cette réflexion sur les autres systèmes de savoir, entamée peu après la seconde guerre mondiale, continue de nos jours. De fait, après l'aspect philosophique, cognitif, épistémologique des premières recherches sur les sciences des autres, une dimension beaucoup plus pragmatique s'exprime aujourd'hui dans l'intérêt pour les savoirs indigènes. L'échec du développement en tant que modèle inadapté, la prise de conscience des conséquences environnementales du productivisme forcené de la révolution agricole ont conduit à développer de nouveaux paradigmes comme celui du développement durable et participatif. Après le rapport Bruntland, le Sommet de Rio, la Convention de la biodiversité, les discussions de la FAO sur les ressources génétiques, le projet de Déclaration des droits des peuples indigènes ont mis à l'agenda le respect et la sauvegarde des savoirs indigènes en tant qu'instrument de gestion. Mais au moment où tous se mettent à inclure les savoirs locaux dans leur discours, si l'on ne veut pas voir dans cette notion une évocation purement rhétorique, ou un des lieux du politiquement et environnementalement correct, il nous paraît important de remonter aux sources. 


\section{BIBLIOGRAPHIE}

Amselle J. L. 2008 - Retour sur « l'invention de la tradition ». L'Homme (185-186). [En ligne], mis en ligne le 29 octobre 2008. URL : http://lhomme.revues.org/index17122.html.

Augé M. 1994 - Pour une anthropologie des mondes contemporains, Paris, Flammarion, 196 p. (Champs Sciences).

Barrau J. 1985 - À propos du concept d'ethnoscience. In Les savoirs naturalistes populaires : actes du séminaire de Sommières, 12 et 13 décembre 1983. Paris, Éditions de la Maison des Sciences de l'Homme : 5-12.

Birkeland C. 2003 - Johannes as a pioneer. Traditional Marine Resource Management and Knowledge Information Bulletin. Special Edition, March 2003 : 18.

Brody H. 1981 - Maps \& Dreams. Indians and the British Columbia Frontier, Long Grove, Illinois, Waveland Press, 294 p.

Callon M., Lascoumes P. \& Barthe Y. 2001 - Agir dans un monde incertain. Essai sur la démocratie technique, Paris, Le Seuil. (La couleur des idées).

Chambers R., Pacey A. \& Thrupp L. 1989 - Farmer First : Farmer Innovation and Agricultural Research. London, Intermediate Technology Publications, $218 \mathrm{p}$.

Charvolin F., Micoud A. \& Nhyart L.K. (Dir.) 2007 - Des sciences citoyennes ? La question de l'amateur dans les sciences naturalistes. La Tour d'Aigues, Éditions de l'Aube, 258 p. (Monde en cours - essais).

Conklin H.C. 1955 - The Relation of Hanunoo Culture to the Plant World. Ph. D. dissertation, Yale University. Ann Arbor, Michigan, University Microfilms Inc., 471 p.

Feit H.A. 1973 - The Ethno-Ecology of the Waswanipi Cree : Or How Hunters Can Manage Their Resources. In Cox B. (Ed.), Cultural Ecology: Readings on the Canadian Indians and Eskimos. Toronto, McClelland and Stewart Limited : 115-125.

Freeman M. (Dir.) 1976 - Inuit Land Use and Occupancy Project: a Report. Ottawa, Minister of Supply and Services Canada, 3 tomes.

Freeman M. 1992 - The nature and utility of traditional ecological knowledge. Northern Perspectives 20 (1): 7-12.

Freeman M. 2011 - Looking back - and looking ahead - 35 years after the Inuit land use and occupancy project. The Canadian Geographer / Le Géographe canadien, 55, issue 1.

Glissant E. 1997 - Traité du Tout-Monde. Paris, Gallimard, 272 p.

Goody J. 1977 - Mémoire et apprentissage dans les sociétés avec ou sans écriture : la transmission du Bagre. L'Homme 17 (1) : 29-52.

Howes M. \& Chambers R. 1980 - Indigenous technical knowledge: analysis, implications and issues. In Brokensha D., Warren D. M. \& Werner O. (Ed.), Indigenous Knowledge Systems and Development. Washington, D.C., University Press of America : 323-334.

Inglis J.T. (Ed.) 1993 - Traditional Ecological Knowledge - Concepts and Cases. IDRC Books, 150 p. 
Johannes R.E. 1981 - Words of the Lagoon : Fishing and Marine Lore in the Palau District of Micronesia. Berkeley, University of California Press, $320 \mathrm{p}$.

Johannes R.E. (Ed.) 1989 - Traditional Ecological Knowledge : A Collection of Essays. Gland, IUCN, 77 p.

Krupnik I. \& Jolly D. (Ed.) 2002 - The Earth is Faster Now : Indigenous Observations of Arctic Environmental Change. Fairbanks, Arctic Research Consortium of the United States (ARCUS), 356 p.

Latour B. 2006 - Nous n'avons jamais été modernes. Essai d'anthropologie symétrique. Paris, La Découverte Poche/Sciences humaines et sociales 26. (1 ère édition 1991, La Découverte, « L'armillaire »).

Lee R.B. \& DeVore I. (Ed.) 1968 - Man the Hunter. Chicago, Aldine Transaction, 415 p.

Lee R.B. \& DeVore I. (Ed.) 1976 - Kalahari Hunter-Gatherers : Studies of the!Kung San and Their Neighbours. Cambridge, MA., Harvard University Press, 408 p.

Lenclud G., 1987 - La tradition n'est plus ce qu'elle était... Terrain (9) : 110-123.

Lévi-Strauss C. 1962 - La Pensée Sauvage, Paris, Plon, 393 p.

Nakashima J. D. 1993 - Astute observers on the sea ice edge : Inuit knowledge as a basis for Arctic co-management. In Inglis J. (Ed.), Traditional ecological knowledge: Concepts and cases. Ottawa, International Development Research Center : 99-110.

Olivier de Sardan J.P. 1991 - Les trois approches en anthropologie du développement. In : Tiers-Monde. 2001, 42 (168) : 729-754. doi : 10.3406/tiers.2001.1546, http://www.persee.fr/web/revues/home/ prescript/article/tiers_1293-8882_2001_num_42_168_1546 [en ligne], [Consulté le 25 avril 2012].

Peeters A. 1979 - Nomenclature and Classification in Rumphius's Herbarium Amboinense. In Ellen R.F. and Reason D. (Ed.), Classifications in their social context. London, Academic Press, 262 p.

Pouillon J. 1998 - Le Cru et le Su, Paris, Seuil, 169 p. (La librairie du xx siècle).

Pouillon J. 1975 - Tradition : transmission ou reconstruction, in Pouillon J. (Ed.) Fétiches sans

fétichismes. Paris, Maspero : 155-173.

Ruddle K. \& Johannes R.E. (Ed.) 1985 - The Traditional Knowledge and Management of Coastal Systems in Asia and the Pacific. Jakarta, Indonesia, UNESCO-ROSTSEA, $313 \mathrm{p}$.

Sahlins M. 1972 - Stone Age Economics. Chicago, Aldine Transaction, 348 p.

Sahlins M. 1976 - Âge de pierre, âge d'abondance. L'économie des sociétés primitives. Jolas T. (Trad.).

Paris, Gallimard, nrf, 409 p. (Bibliothèque des Sciences Humaines).

SPC 2003 - Tributes to Bob Johannes. Traditional Marine Resource Management and Knowledge Information Bulletin. Special Edition, March 2003, $32 \mathrm{p}$.

Speck F.G. 1923 - Reptile lore of the Northern Indians. Journal of American Floklore, 36, nº141.

\section{NOTES}

1. Cf. par exemple www.un.org/esa/socdev/unpfii/fr/ : Permanent Forum on Indigenous Issues to Meet at the United Nations, New York, 13-24 May 2002.

2. Dans le chapitre V de son rapport du 30 septembre 1983 pour la Commission des droits de l'homme du Conseil économique et social des Nations Unies sur la discrimination envers les populations autochtones, Jose Martinez Cobo, rapporteur spécial, conclut qu'aucun critère objectif ne pouvant être définitivement établi, sont donc autochtones ceux que les populations 
autochtones elles-mêmes considèrent comme tels selon leur propre auto-définition

(http://www.un.org/esa/socdev/unpfii/documents/MCS_xxi_xxii_e.pdf)

3. Cf. http://www.unesco.org/new/en/natural-sciences/priority-areas/links/

4. Du moins fut-il le premier à mettre en valeur une collaboration d'égal à égal. Il faudrait réécrire l'histoire des savoirs locaux en relisant les grands explorateurs qui travaillaient tous avec des informateurs locaux dont ils dépendaient largement. C'est par exemple le cas de von Humboldt au XVIII ${ }^{\mathrm{e}}$ siècle. Dès le XVII ${ }^{\mathrm{e}}$ siècle G.E. Rumpf, employé par la Dutch East India Company en Indonésie dans l'île d'Ambon, de 1653 à sa mort en 1702, fit œuvre d'ethnobotaniste (Peeters 1979). La traduction de son ouvrage magistral en latin, en 6 volumes, avec des notes et commentaires, fut entreprise après sa mort par le botaniste Burman qui prépara le texte et traduisit l'Herbarium Amboinense. Non seulement Rumpf donne les noms vernaculaires, mais il note également les usages et croyances locales, ainsi que beaucoup des catégories de plantes distinguées par les taxinomies locales.

5. Lire aussi dans Johannes (1989: 35) le récit épique qu'il fait de la révélation du savoir local. Daelbai, un pêcheur de Palau demande à Johannes de quelle couleur il voit le sable. Quand celuici répond blanc, Daelbai lui déclare alors qu'à 4 heures ce sera noir : «à 4 heures c'était noir - de poissons, des milliers et des milliers de mojaras (Gerres oblongus) ».

\section{RÉSUMÉS}

Nous tenterons ici une histoire anthropologique de la notion de "savoirs locaux", depuis les premiers écrits dans les années 1950 qui se sont intéressés aux savoirs botaniques ou zoologiques des peuples «traditionnels " jusqu'à l'explosion d'un intérêt pour ces savoirs qui réunit des acteurs aussi disparates que la Banque Mondiale, les ONG de conservation et de développement, les gouvernements, les gestionnaires de la biodiversité, sans oublier les principaux acteurs, peuples autochtones ou groupes spécialisés locaux.

En développant une histoire des différents réseaux qui ont participé à l'élaboration du concept de savoirs locaux, savoirs écologiques traditionnels ou autochtones, nous repérerons les précurseurs en les replaçant dans leur contexte heuristique. Nous nous intéresserons aussi aux tendances plus récentes, depuis l'inscription des savoirs locaux dans plusieurs conventions internationales, en particulier la Convention sur la diversité biologique. Nous examinerons enfin la portée épistémologique de l'avènement de savoirs « locaux » qui se confrontent ou se conjuguent avec les savoirs scientifiques et les savoirs « profanes».

In this paper, we will attempt an anthropological history of the concept of "local knowledge", from the first research dedicated to the botanical or zoological knowledge of "traditional" peoples carried out in the 50's, to the outburst of interest on behalf of actors as disparate as the World Bank, conservation and development NGOs, governments, biodiversity managers, not to mention the main stakeholders, i.e. indigenous peoples and local specialised groups.

Through the history of different networks that contributed to developing the concept of local knowledge, traditional or indigenous ecological knowledge, we will highlight precursors and replace them in their heuristic context. We will also consider more recent trends, since the inclusion of traditional knowledge in several international conventions, particularly the Convention on Biodiversity. We will finally examine the epistemological impact of the combination of local knowledge and scientific and lay knowledge. 
INDEX

Keywords : local knowledge, traditional ecological knowledge, indigenous knowledge

Mots-clés : savoirs locaux, savoirs écologiques traditionnels, savoirs autochtones

\section{AUTEUR}

MARIE ROUÉ

UMR 7206 MNHN-CNRS-Université Paris Diderot

Éco-Anthropologie et Ethnobiologie

Muséum national d'histoire naturelle

Département Hommes, Natures, Sociétés

case postale 135

57 rue Cuvier, 75005 Paris (France)

roue@mnhn.fr 